\title{
Faster raster matrix-assisted laser desorption/ionization mass spectrometry imaging of lipids at high lateral resolution
}

Citation for published version (APA):

Barre, F., Rocha, B., Dewez, F., Towers, M., Murray, P., Claude, E., Cillero-Pastor, B., Heeren, R., \& Siegel, T. P. (2019). Faster raster matrix-assisted laser desorption/ionization mass spectrometry imaging of lipids at high lateral resolution. International Journal of Mass Spectrometry, 437, 38-48. https://doi.org/10.1016/j.ijms.2018.09.015

Document status and date:

Published: 01/03/2019

DOI:

10.1016/j.ijms.2018.09.015

Document Version:

Publisher's PDF, also known as Version of record

\section{Document license:}

Taverne

Please check the document version of this publication:

- A submitted manuscript is the version of the article upon submission and before peer-review. There can be important differences between the submitted version and the official published version of record.

People interested in the research are advised to contact the author for the final version of the publication, or visit the DOI to the publisher's website.

- The final author version and the galley proof are versions of the publication after peer review.

- The final published version features the final layout of the paper including the volume, issue and page numbers.

Link to publication

\footnotetext{
General rights rights.

- You may freely distribute the URL identifying the publication in the public portal. please follow below link for the End User Agreement:

www.umlib.nl/taverne-license

Take down policy

If you believe that this document breaches copyright please contact us at:

repository@maastrichtuniversity.nl

providing details and we will investigate your claim.
}

Copyright and moral rights for the publications made accessible in the public portal are retained by the authors and/or other copyright owners and it is a condition of accessing publications that users recognise and abide by the legal requirements associated with these

- Users may download and print one copy of any publication from the public portal for the purpose of private study or research.

- You may not further distribute the material or use it for any profit-making activity or commercial gain

If the publication is distributed under the terms of Article $25 \mathrm{fa}$ of the Dutch Copyright Act, indicated by the "Taverne" license above, 


\title{
Faster raster matrix-assisted laser desorption/ionization mass spectrometry imaging of lipids at high lateral resolution
}

\author{
Florian Barré $^{\mathrm{a}, 1}$, Beatriz Rocha ${ }^{\mathrm{a}, 1}$, Frédéric Dewez $^{\mathrm{a}}$, Mark Towers ${ }^{\mathrm{b}}$, Paul Murray ${ }^{\mathrm{b}}$, \\ Emmanuelle Claude ${ }^{b}$, Berta Cillero-Pastor ${ }^{a}$, Ron Heeren ${ }^{a}$, Tiffany Porta Siegel ${ }^{a, *}$ \\ ${ }^{a}$ Maastricht MultiModal Molecular Imaging (M4I) Institute, Division of Imaging Mass Spectrometry, Maastricht University, Universiteitssingel 50, 6229 ER, \\ Maastricht, the Netherlands \\ ${ }^{\mathrm{b}}$ Waters Corporation, Wilmslow, United Kingdom
}

\section{A R T I C L E I N F O}

\section{Article history:}

Received 9 April 2018

Received in revised form 31 August 2018

Accepted 13 September 2018

Available online 18 September 2018

\section{Keywords:}

Mass spectrometry imaging

Rastering imaging

Cartilage

Cellular imaging

MALDI imaging

Ion mobility spectrometry imaging

\begin{abstract}
A B S T R A C T
The clinical use of mass spectrometry imaging (MSI) is rapidly growing, and applications are expanding. Crucially, the analysis of clinical cohorts requires a combination of higher analytical throughput and higher lateral resolution. Here, we demonstrate the benefits of an improved design of a prototype MALDI source (referred as "uMALDI") mounted on a SYNAPT HDMS G2-Si mass spectrometer - widely used by the MSI community. The uMALDI source allows for imaging of lipids on histologically well documented rat brain sections at a lateral resolution of $15 \mu \mathrm{m}$ without oversampling. We also investigate the fast rastering capabilities of the platform enabled by a new web-based MS control program (i.e. "WREnS" Waters Research Enabled Software). The combination of the uMALDI and WREnS allows high spatial resolution images at acquisition rates up to 20 pixels per second. Additionally, we demonstrate the capability of this platform to characterize the lipid distribution of osteoarthritic (OA) cartilage at the cellular level, which highlights potential benefit for broader application in clinical context. Finally, we prove that ion mobility separation of isobaric lipid species is maintained and images can be acquired at a rate of 10 scans per seconds. This renders a unique ion mobility mass spectrometric imaging platform with high acquisition rates and high spatial resolution.
\end{abstract}

(C) 2018 Elsevier B.V. All rights reserved.

\section{Introduction}

Mass spectrometry imaging (MSI) is an analytical technique that is able to determine the relative abundance and spatial distribution of a large variety of biomolecules, such as lipids, peptides, proteins, metabolites and amino acids [1-3], in tissue sections without the use of labelling or staining [4]. MSI molecular data can be directly correlated with anatomical tissue features [5,6] and in order to have an accurate integration between histology and the molecular localization, high spatial resolution is needed. The spatial resolution obtained with matrix-assisted laser desorption/ionization (MALDI)-MSI is improving with the development of high spatialresolution MS instruments [7,8]. This enables exploration of cellular molecular signatures required for many pathology applications,

\footnotetext{
* Corresponding author.

E-mail address: t.porta@maastrichtuniversity.nl (T. Porta Siegel).

1 Equal contribution.
}

such as the characterization of intratumor molecular heterogeneity [9].

MSI has already demonstrated its impact on several clinical applications, such as biomarker discovery, tissue or patient classification and drug monitoring, just to name a few [10]. MALDI-MSI has been used to investigate the pathogenesis of different cancers and neurodegenerative diseases. In rheumatology, MALDI-MSI has been performed on articular cartilage, synovium and bone to increase the understanding of articular destruction and to characterize diagnostic markers for rheumatic diseases such as osteoarthritis (OA) and rheumatoid arthritis [11,12].

It is crucial to improve the overall analytical workflow with robust sample preparations, faster acquisition rates and augmented information content, all needed to meet clinical needs. The time it takes to acquire each high information content dataset remains one of the main limitations of MSI. In fact, certain measurements can take up to several days, dependent on the acquisition area and image resolution required to answer a specific question. The analysis time is mainly determined by the type of mass analyzer, the acquisition mode used and the number of pixels 
that need to be acquired [13]. The design of experiment strongly influences the quality of the image generated. The choice of the size of the pixel/voxel defined to produce the molecular image is a key element of the experiment. Most commonly, the microprobe mode is used to acquire MS images and one pixel corresponds to one MS experiment (e.g. one mass spectrum in full scan mode). There are two ways to acquire an image dataset: the discrete mode and the rastering mode. During discrete or spot-by-spot imaging, the stage moves in discrete steps and the pulsed laser is fired when the sample is in the designated position. The rastering mode is an approach where the sample stage is moved at a constant speed while the pulsed laser is continuously firing such that each laser pulse interacts with a slightly different area on the surface [14]. The continuous laser raster mode has shown to significantly increase the speed of the acquisitions compared to the discrete mode. Rastering reduces image acquisition rates by a factor or 2.5-5 while simultaneously improving sensitivity and spatial resolution [15]. The combination with high-repetition rate lasers (e.g. $10 \mathrm{kHz}$ solid state neodymium-doped yttrium aluminum garnet (Nd:YAG), $\mathrm{Nd}$ :YLF, or Nd:YVO4) and continuous raster considerably promote faster imaging data acquisition. Accordingly, high data acquisition rates of 50 pixels per second have been recently achieved using a new high-speed MALDI-time-of-flight (TOF) MSI system with a $10 \mathrm{kHz} \mathrm{Nd}$ :YAG laser [16]. This platform offered the great benefit to use volatile matrices within high-vacuum systems for MALDIMSI, which was not possible before. In addition, imaging lipid distribution of a sagittal rat brain tissue section can be obtained in 10 min with an acquisition rate of 30 pixels per second employing a laser repetition rate of $5 \mathrm{kHz}$ in continuous raster mode [17]. These technical developments offer the possibility to analyze a substantial number of samples from large patient cohorts in a short period of time and enable the translational implementation of MSI in clinical and preclinical studies.

Gas-phase ion mobility spectrometry (IMS) technologies have been coupled to MSI imaging and allow rapid structural separation of molecular ions. This approach improves the detection of low abundance ions and the separation of isobaric molecular species that have a different collision cross-section. This augments the analytical information content of the MSI dataset and enables a more accurate determination of the spatial distribution of different molecular ions [18].

Here, we describe a prototype of an improved MALDI source (referred as "UMALDI") mounted on a SYNAPT HDMS G2-Si mass spectrometer. The uMALDI source allowed for imaging of lipids on well histologically documented rat brain sections at a routine lateral resolution of $15 \mu \mathrm{m}$ without oversampling. The uMALDI source was used in combination with the Waters Research Enabled Software (WREnS) which enables data acquisition rates up to 20 pixels per second. The improved quality of the imaging data generated by the combination of the new design of the source and the new software is discussed. Additionally, we demonstrate the potential of this platform to characterize the lipid distribution of osteoarthritic (OA) cartilage at the cellular level. Finally, we successfully coupled MALDI-MSI and WREnS with ion mobility, which renders a unique ion mobility imaging platform with fast acquisition rates and high spatial resolution.

\section{Material and method}

\subsection{Chemicals and reagents}

Norharmane and $\alpha$-Cyano-4-hydroxycinnamic acid (CHCA) were purcharsed from Sigma-Aldrich (Gillingham, Dorset, U.K.). Chloroform $\left(\mathrm{CHCl}_{3}\right)$, methanol $(\mathrm{MeOH})$ and ethanol (EtOH) were purchased from Fisher Scientific (Loughborough, Leicestershire,
U.K.). Penicillin / streptomycin (P/S) were purchased from Thermo Fisher Scientific (Waltham, MA, U.S.A.). Ammonium acetate was purchased from AMRESCO (Solon, OH, U.S.A.).

\subsection{Tissue collection and preparation}

Healthy Wistar Han rat and mouse brain tissue samples were provided by the group of General Surgery, Maastricht University Medical Center (MUMC+), Maastricht, The Netherlands. Organ extraction protocol complied with the Dutch Animal Experimental Act and was approved by the Animal Experimental Committee of MUMC + . Articular cartilage was harvested from knee joints derived from patients undergoing arthroplasty. Anonymous use of redundant tissue for research purposes is part of the standard treatment agreement with patients in the UMCU and materials are used according to the guideline 'good use of redundant tissue for clinical research' constructed by the Dutch Federation of Medical Research Societies (http://www.federa.org/code-goed-gebruikvan-lichaamsmateriaal-2011). OA articular cartilage explant was cut into $5 \mathrm{~mm}$-discs using a sterile biopsy punch. After extensive washes with PBS 1\% penicillin/streptomycin and ice-cold ammonium acetate buffer ( $150 \mathrm{mM}, \mathrm{pH} 7.3)$, cartilage discs were snap-frozen in liquid nitrogen, and stored at $-80^{\circ} \mathrm{C}$ until sectioning. Frozen tissues were then cryo-sectioned at $-20^{\circ} \mathrm{C}$ using a cryo-microtome (Leica Microsystems, Wetzlar), at $10 \mu \mathrm{m}$ for the rat brain sample and $12 \mu \mathrm{m}$ thickness for the cartilage sample. Both samples where thaw-mounted onto regular non-conductive glass slides and stored at $-80^{\circ} \mathrm{C}$ until further analysis.

\subsection{MALDI matrix application}

Just before analysis, the tissue sections were rapidly dried at room temperature using a gentle flow of nitrogen gas $(30 \mathrm{~s})$. A norharmane solution $\left(7 \mathrm{mg} / \mathrm{mL}\right.$ in $\mathrm{CHCl}_{3}: \mathrm{MeOH}, 2: 1$, v:v) was sprayed on rat brain and cartilage tissue sections using the TMSprayer from HTX-Technologies (HTX Technologies, LLC, North Carolina, USA) for positive mode analysis. 12 matrix layers were applied at a flow rate of $0.120 \mathrm{~mL} / \mathrm{min}$. The spray nozzle was heated up at $30^{\circ} \mathrm{C}$, and the velocity of the nozzle was set at $1200 \mathrm{~mm} / \mathrm{min}$ (nozzle height: $40 \mathrm{~mm}$ ). On alternating matrix passes, the spray pattern was changed by an angle of $90^{\circ}$ and adjusted with a line spacing of $3 \mathrm{~mm}$. A waiting time of $30 \mathrm{~s}$ was set between each layer in order to let the matrix dry to reduce the size of the matrix crystals.

Mouse brain sections were prepared using the same sprayer. 8 layers of a CHCA solution ( $5 \mathrm{mg} / \mathrm{mL}$ in acetonitrile:water, $70: 30$ ) were deposited at $85^{\circ} \mathrm{C}$ for negative mode analysis. The line spacing was set at 2 with a velocity of $1300 \mathrm{~mm} / \mathrm{min}$ and a flow rate of $0.200 \mathrm{~mL} / \mathrm{min}$ in order to achieve homogeneous matrix coverage.

\subsection{Mass spectrometry imaging (MSI) experiments}

\subsubsection{General settings}

Mass spectrometry acquisitions were performed on two independent MALDI SYNAPT High Definition mass spectrometer (HDMS) G2-Si systems (Waters Corporation, Manchester, U.K.). Both systems were tuned to conduct MALDI-MSI experiments and equipped with a $2.5 \mathrm{kHz}, \mathrm{Nd}$ :YAG laser, producing $25 \mathrm{~nJ}$ pulses at a wavelength of $355 \mathrm{~nm}$. The first MS system was equipped with a first generation Waters MALDI source. The second system was equipped with a prototype source (referred in this manuscript as "uMALDI"). The uMALDI source consists of an $\mathrm{x}-\mathrm{y}$ stage with $1 \mathrm{~mm}$ pitch lead screws, an ion guide and extraction electrode assembly. Loading and unloading samples is achieved by opening and closing a door, carrying the sample stage. The uMALDI beam path is adjusted using two dielectric mirrors, and passes through a variable transmission neutral density wheel, and fused silica $75 \mathrm{~mm}$ 
focal length best form lens before being directed onto the MALDI sample plate. The lens is mounted on a linear translation stage, allowing the diameter of the beam incident on the sample plate to be varied from around $15 \mu \mathrm{m}$ to $150 \mu \mathrm{m}$. An additional neutral density filter can be inserted into the beam path to further attenuate the laser intensity, when operating with the laser beam tightly focused on the sample.

The samples were optically scanned using a flatbed scanner to produce a digital image for future reference. This image was then imported into the MALDI imaging pattern creator software (High Definition Imaging (HDI) v1.4 software, Waters) to define the region to be imaged. Acquisitions were performed on both instruments in "sensitive MS mode" using positive or negative ionization modes for lipid detection. Instrument calibration was performed in both positive and negative ion mode using a standard calibration mixture of red phosphorous [19].

\subsubsection{Spot-to-spot imaging}

The spot-to-spot imaging experiments on rat cerebellum were performed at 2.5 (at $45 \times 45 \mu \mathrm{m}^{2}$ spatial resolution) and 4 pixels per second (at $15 \times 15 \mu \mathrm{m}^{2}$ spatial resolution) with a laser frequency of $1000 \mathrm{~Hz}$ over the mass range $m / z 200-1000$. The laser pulse energy was optimized for $15 \times 15 \mu \mathrm{m}^{2}$ spatial resolution experiments corresponding to a value of 240 (arbitrary units, a.u.) in the instrument. On the other hand, the laser pulse energy employed in the experiments conducted at $45 \times 45 \mu \mathrm{m}^{2}$ spatial resolution was optimized at 310 (a.u.) in the mass spectrometer. The quadrupole profile was set in automatic profile mode.

\subsubsection{Effect of acquisition speed at a fixed pixel size}

$12 \mu \mathrm{m}$ thick mouse sagittal brain sections mounted on nonconductive glass slides were prepared by spray coating with recrystallized CHCA. $5 \mathrm{mg} / \mathrm{ml}$ CHCA in 70:30 water:acetonitrile $+0.2 \%$ TFA was applied using the Suncollect sprayer (from Sunchrom). An initial 2 layers were sprayed at $15 \mu \mathrm{l} / \mathrm{min}$ followed by 33 layers at $20 \mu \mathrm{l} / \mathrm{min}$ - line spacing was $1.5 \mathrm{~mm}$ using speed medium 1 , the nebulising gas was set to 1.75 bar.

\subsubsection{Continuous raster imaging}

Half mouse brains were measured using the uMALDI source with the following parameters: mass range $m / z 400-1000$ with a fixed quadrupole profile at $m / z 500$ to avoid matrix peaks in the low mass range. The laser pulse energy was also optimized in the same way as in the spot-to-spot experiments. For continuous raster measurements, we employed a laser pulse energy value of 240 (a.u.) and a laser repetition rate of $1000 \mathrm{~Hz}$.

For cartilage analysis, the mass range was set to $m / z$ 400-1150 with an automatic mode profile for the quadrupole with a laser pulse energy of 300 in order to get signal from the tissue. The analysis was performed at a speed of 20 pixels per second and with a laser repetition rate of $1000 \mathrm{~Hz}$.

\subsubsection{Ion mobility spectrometry}

Ion mobility separation was performed over the mass range $m / z$ 400-1000 (lipids) using nitrogen as a drift gas with a flow rate of $90 \mathrm{~mL} / \mathrm{min}$. Different transfer T-wave velocities were tested, ranging from 215 to $245 \mathrm{~m} / \mathrm{s}$ to assess aliasing artefacts between the transfer cell and the TOF pusher. The TRAP DC entrance was set to $0 \mathrm{~V}$ and the wave height was set to $40 \mathrm{~V}$. The velocity of IMS wave was used to divide the ions over the total $200 \mathrm{~ms}$. The start velocity was set at $1300 \mathrm{~m} / \mathrm{s}$ and the end velocity at $300 \mathrm{~m} / \mathrm{s}$.

\subsection{Lipid assignments}

Tandem mass spectrometry was performed directly on different tissue sections in both positive and negative ion modes. Lipid ion precursors were selected within a 1 Da selection window and fragmented using 10-40 V collision energy applied in the ion trap cell. The obtained spectra were processed using MassLynx software. The MS/MS fragmentation pattern for each precursor mass was used to identify the lipids of interest and to assign them by manual comparison with lipid databases (www.lipidmaps.org) and relevant literature.

\subsection{Staining of the tissue sections}

Hematoxylin-eosin (H\&E) staining was performed on the same brain tissue section used for MALDI-MSI measurements. Following MALDI-MSI experiments, the slides were first dipped in a 70\% EtOH solution for $5 \mathrm{~min}$ in order to remove the residual matrix. Then, the $\mathrm{H} \& \mathrm{E}$ staining was carried out as follow: the tissue sections were re-hydrated in water for $1 \mathrm{~min}$; stained in hematoxylin staining for 3 min; washed under running tap water for $3 \mathrm{~min}$; stained in eosin for $30 \mathrm{~s}$; washed under running tap water for $3 \mathrm{~min}$; immersed in $100 \%$ EtOH for $1 \mathrm{~min}$ and finally transferred into xylene for $2 \mathrm{~min}$. An Entellan mounting medium was applied to the slides and carefully covered with a coverslip. The samples were dried at room temperature.

Safranin-O and Fast Green FCF staining was performed on cartilage tissue sections by removing the matrix by immersion in EtOH for $5 \mathrm{~min}$. The sections were stained using hematoxylin ( $8 \mathrm{~min})$ to stain the nuclei of the chondrocytes, rinsed in running tap water for $5 \mathrm{~min}$ and stained with Safranin-O 0.1\% solved in ultra-pure water for $4 \mathrm{~min}$. After a short rinse in running tap water, the sections were immersed in Fast Green FCF 0,1\% solved in ultra-pure water for $4 \mathrm{~min}$. Short rinses in EtOH $96 \%$ and $100 \%$ were used before to transfer the sections to xylene for $10 \mathrm{~min}$. The optical images were acquired with high resolution using the Mirax Desk Slide Scanner2.6 Software (Zeiss, Gottingen, Germany).

\subsection{Software}

MassLynx 4.1 (Waters) was used for instrument control and data acquisition in conventional discrete (i.e. "spot-by-spot") acquisition mode available on the SYNAPT HDMS G2-Si. The rastering acquisition mode was facilitated by Waters Research Enabled Software (WREnS). The WREnS interface is an independent, web-based program that applies user written scripting (i.e. $C$ in the present study) in real-time directly to the EPC.

The HDI processed data was subsequently normalized against the total ion count using an in-house script. HDI software (Waters) was used for post-acquisition processing and visualization of MS imaging data generated.

\section{Results and discussion}

Here, we first describe the performance of a prototype uMALDI platform for high throughput and high spatial analysis of lipids. For this purpose, the first experiments rely on the investigation of lipids in rat brain tissues and more specifically from the cerebellum region, which consists of well-defined histological features andwhich is well documented in the literature for comparison. 

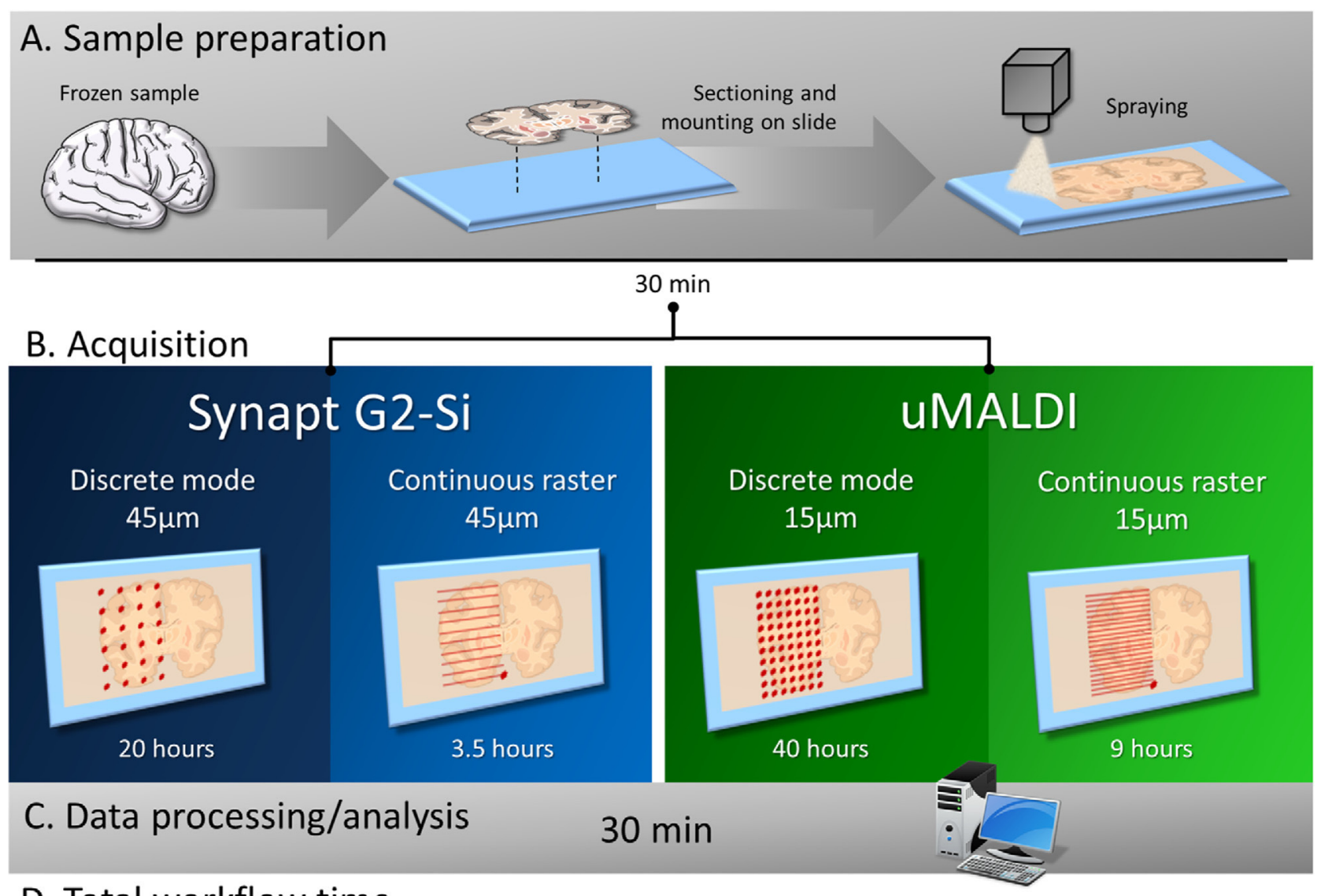

\section{Total workflow time}
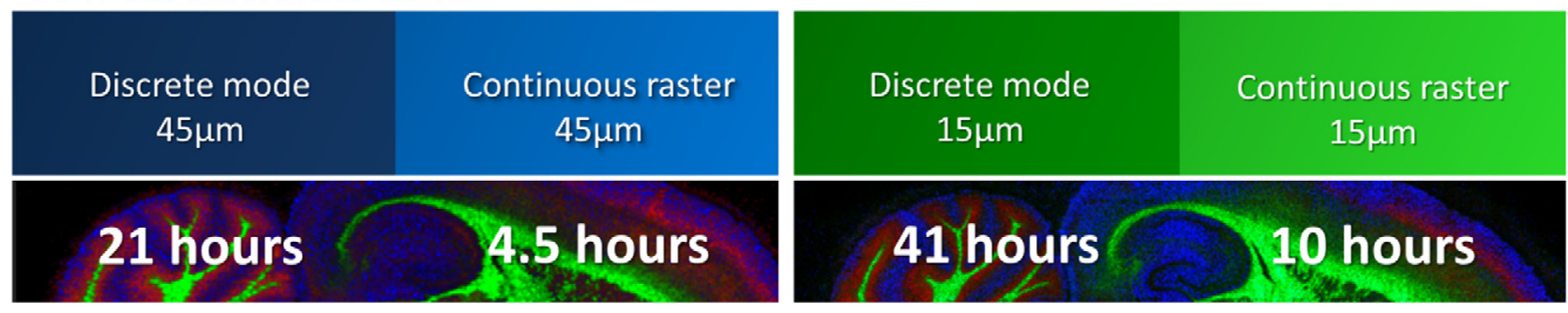

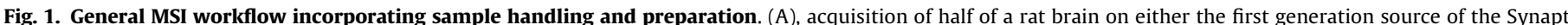

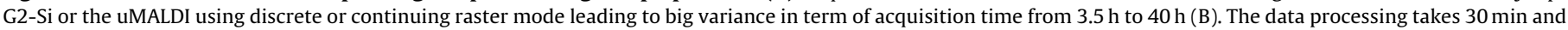
allows spatial visualization of compounds of interest (C). According to the chosen workflow, the overall analytical time can vary between $4.5 \mathrm{~h}$ to $41 \mathrm{~h}$ (D).

Then, we assess the performance of the uMALDI MS system used in combination with the Waters Research Enabled Software (WREnS) to allow a fast raster acquisition methodology. These new developments lead to faster acquisitions of higher definition images (Fig. 1). Fig. 1 compares and contrasts the performance at maximum spatial resolution of the two systems.

\subsection{New uMALDI source design allows high spatial resolution imaging at high speed}

MS images were first acquired in conventional discrete (i.e. "spot-by-spot") acquisition mode to compare the performances of the first generation MALDI and new UMALDI design of the SYNAPT HDMS G2-Si source in term of both spectral and image quality. The first set of experiments was conducted on both instruments with an effective laser spot diameter of $45 \mu \mathrm{m}$ and images were generated with pixel size of $45 \times 45 \mu \mathrm{m}^{2}$. It is noteworthy that $45 \mu \mathrm{m}$ was the minimum laser spot size that could be reached when the laser is attenuated on the first generation MALDI system. The new optical system of the uMALDI enables a more precise and fine tuning of the focus of the laser down to an effective spot size of $15 \mu \mathrm{m}$.

Fig. 2 displays three MALDI-MS images based on the distribution of the ions $\mathrm{m} / \mathrm{z} 769.56$ corresponding to sphingomyelin [SM (d18:1_18:0+K] ${ }^{+}, m / z 826.57$ and $m / z \quad 844.52$ identified as phosphatidylcholines $\left[\mathrm{PC}\left(18: 0 \_18: 1\right)+\mathrm{K}\right]^{+}$and $\left[\mathrm{PC}\left(18: 1 \_22: 6\right)+\mathrm{K}\right]^{+}$, respectively. The species that were selected are specifically located in the white matter, grey matter and granular area of the rat cerebellum, respectively. All compounds were detected in the positive ion mode and identified using MS/MS fragmentation information (see Supplementary Table S1).

When looking at single pixel spectra acquired in spot-to-spot mode, we observed a higher intensity for lipids using the uMALDI source (Fig. 2A and B) in a comparison of the $45 \times 45 \mu \mathrm{m}^{2}$ spatial resolution experiments in positive ion mode. We acknowledge the fact that experiments were conducted on two independent instruments, which were both optimized to operate at their maximum performance (different laser energies and quadrupole profiles tested). Nonetheless, in the conditions described above, additional peaks are observed in the region $m / z 600-900$, corresponding to 


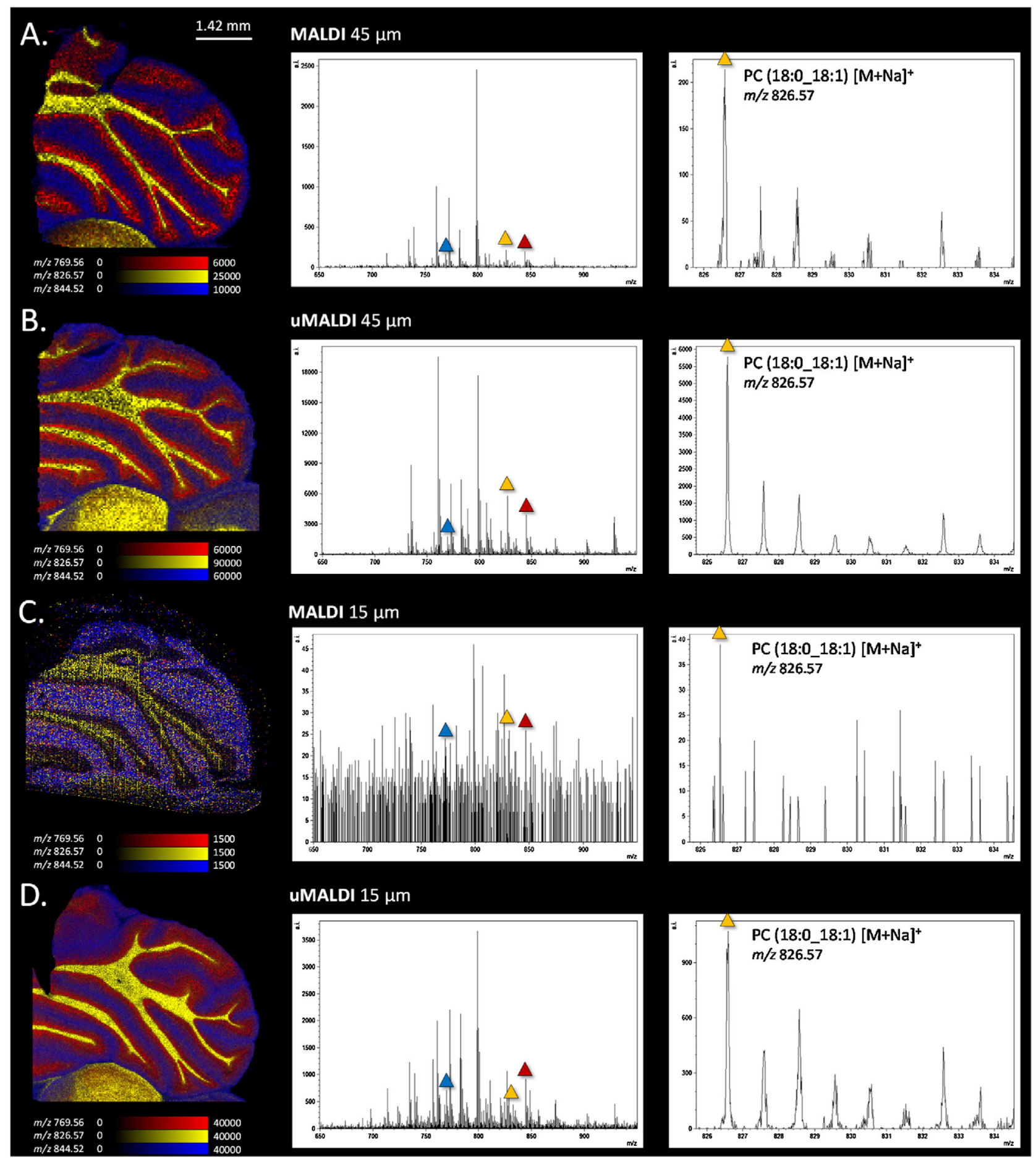

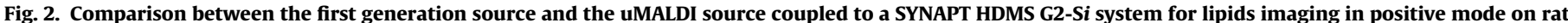

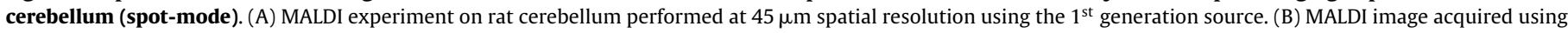

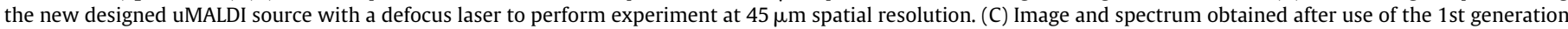
source at $15 \mu \mathrm{m}$ spatial resolution. (D) uMALDI image at $15 \mu \mathrm{m}$ using a focus laser and spectrum extracted from the data.

glycerophospholipids, and with higher signal-to-noise $(\mathrm{S} / \mathrm{N})$ ratio for single pixel spectrum. The $\mathrm{S} / \mathrm{N}$ ratio of the signal obtained for $m / z 826.57$ was more than doubled using the uMALDI (S/N: 24) compared to the 1 st generation MALDI (S/N: 10$)$ at $45 \mu \mathrm{m}$ spatial resolution. This difference can be explained by the absence of oversampling, which has been described earlier to be detrimental to the analysis quality and confirmed here [20]. The modified sample stage allows for a faster but also for more accurate motion which prevents any oversampling during the acquisition, even when acquiring the data in a spot-by-spot basis where oversampling between adjacent pixels can be designed into the experiment. Indeed, the finer pitch on the lead screw from $1.5 \mathrm{~mm}$ to $1 \mathrm{~mm}$ per rotation allows the stage to move in smaller increment. Therefore, when the pitch of the pixels is comparable to the diameter of the laser burn, there is less chance of the burn overlapping with a previous or subsequent pixel.

A second set of experiments was acquired to generate images with pixel size of $15 \times 15 \mu \mathrm{m}^{2}$. For this purpose, as mentioned 

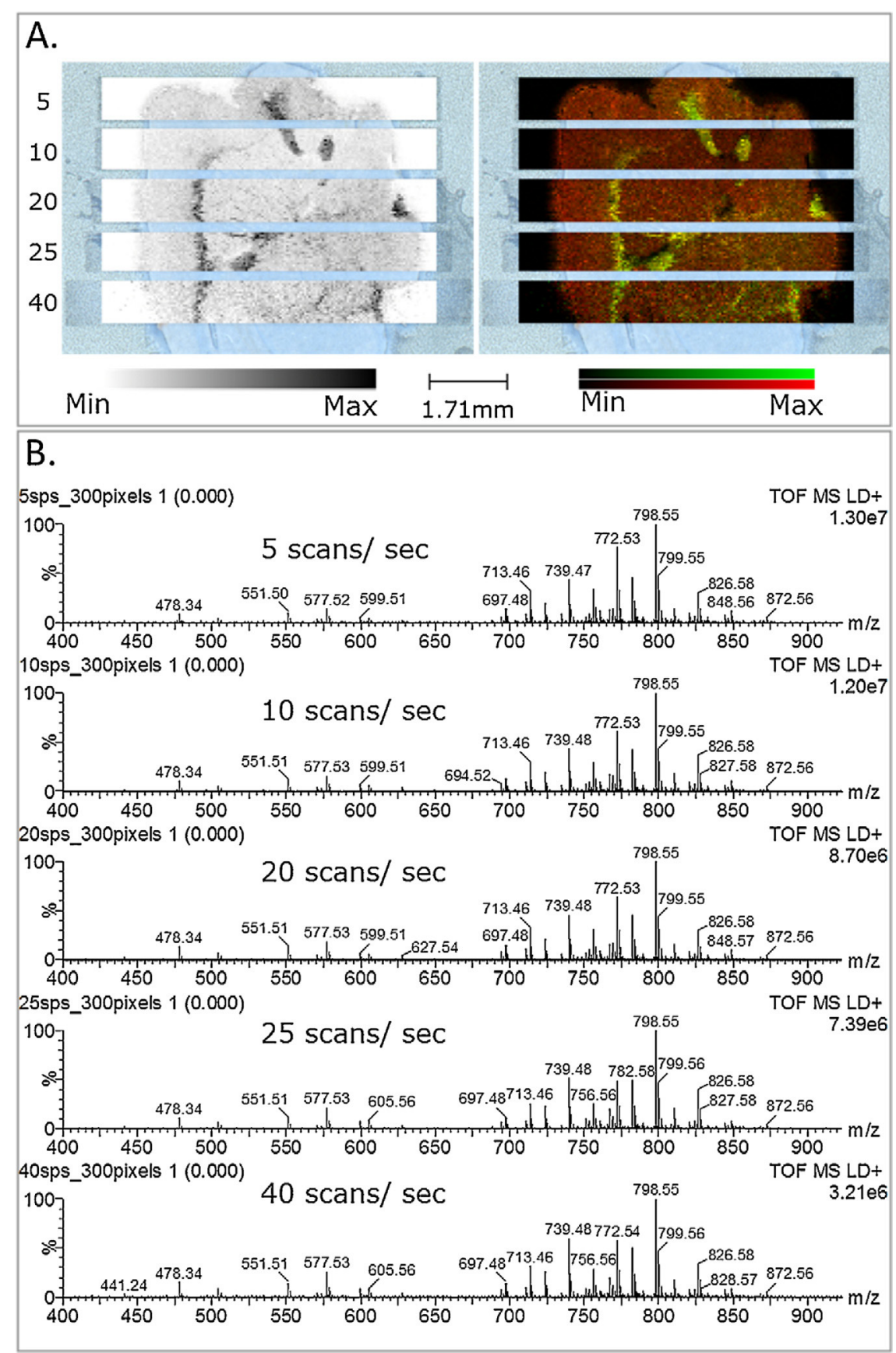

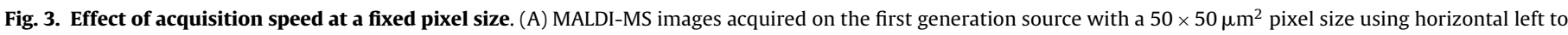

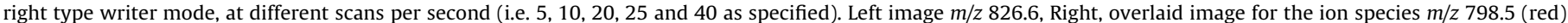

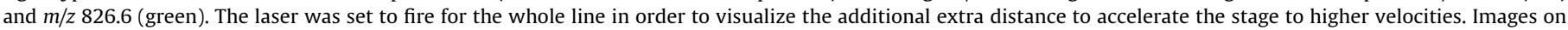

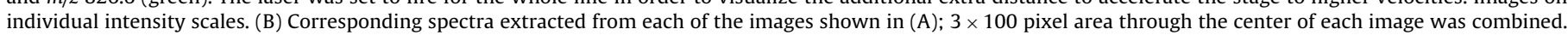

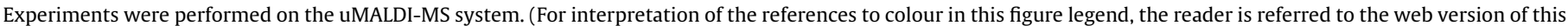
article.)

above, the actual smallest laser spot diameter that could be achieved on the 1 st generation MALDI source was $45 \mu \mathrm{m}$. The experiments on the new uMALDI source were conducted with a focused laser and effective spot diameter of $15 \mu \mathrm{m}$. With the old design and since the actual size of the area sampled is three times the actual size of the sample stage step of $15 \mu \mathrm{m}$ and output pixel displayed, a complete loss of sensitivity is observed- explained by complete oversampling leading to a really poor $\mathrm{S} / \mathrm{N}$ ratio for $\mathrm{m} / \mathrm{z}$ 826.57 (S/N: 1.5) (Fig. 2C). On the other hand, the MALDI-MS data that were acquired with a focused laser on the UMALDI source were of better quality compared with the first generation source obtain- ing a $\mathrm{S} / \mathrm{N}$ ratio of 10 for $\mathrm{m} / \mathrm{z} 826.57$ (Fig. 2D) with a lipid profile which can be compared to the 1st generation MALDI lipid profile acquired at $45 \mu \mathrm{m}$ spatial resolution. When looking at the mean spectra extracted from the tissues, we observed higher relative intensities using the uMALDI source both at 15 or $45 \mu \mathrm{m}$ spatial resolutions (Figure S2) as well as better S/N (Table S2).

The new design of the uMALDI source resulted in an enhancement of the image quality and information content without oversampling. The source is equipped with a high precision stage to take optimal advantages of the improved spatial resolution offered by the new optical system. Both improved sample stage motion 
accuracy and routing laser focus offered a better visualization of the cerebellum structures at a spatial resolution as small as $15 \mu \mathrm{m}$. The new uMALDI source design additionally includes new features that improve source handling and reducing down time. These new features include an easier access to the ion guide (i.e. hexapole region) compared to the first generation source, and allows a cleaning procedure without the need to vent the instrument. For MALDI-MSI users, this is crucially important as the ion optical region has to be cleaned quite often as a result from contamination by MALDI matrices. As we mentioned above, the optimized uMALDI design provided an additional gain in speed compared to the 1 st generation source, since the image acquisition using uMALDI in discrete and continuous raster mode were 1.4 and 4 times faster, respectively. This performance at short time scales could be compatible with clinical practice accelerating the introduction of MSI into the clinic.Other methods that offer higher spatial resolution in MALDIMSI exist [8,21], but they also increase the number of pixels that are acquired which results in acquisition times that are clinically not usable. As an example, going from 15 to $1 \mathrm{um}$ spot size with all parameters equal would result in a 225 -fold increase of acquisition time.

\subsection{Fast raster imaging using a continuously moving sample stage}

Here, we evaluate an alternative acquisition method, involving continuous movement of the sample carrier plate while maintaining pixel synchronization. Scaling the stage speed, laser beam diameter and desired pixel size ensures that the acquisition rate is independent of image resolution. The sample stage movement and laser were controlled using the Waters Research Enabled Software (WREnS) to achieve the higher speed continuous raster imaging mode. Acquired data were pre-processed using a Perl script to allow processing and visualization in Waters High Definition Imaging software (HDI) 1.4 .

Our first investigations were to assess the effect of imaging directionality and continuous acquisition speed on the image quality. WREnS allows direct control of certain elements of the instrument functionality (such as sample stage position) via the input of commands in a script based format. Scripts were created to allow acquisition in both a continuous raster "typewriter" motion as well as a "serpentine" mode, with either a horizontal or vertical directionality [17]. An assessment of the effect of image acquisition direction has been made (see Figure S3). If it can be observed that whilst there are some aberrations due to minor synchronicity effects, the direction of acquisition has no significant effect on the quality or sensitivity of the data (Fig. S3A and S3B; acquired in typewriter at 20 scans per second). These results mean that: i) a serpentine raster, which decreases acquisition time by removing the carriage return is a viable option, as seen in figure S3C and D, and ii) an appropriate direction of acquisition can be selected based on the orientation of the tissue section - which was not possible in previous versions of the software. In Figure S3D the selection of a vertical direction resulted in an $8 \%$ decrease in acquisition time compared to Figure S3C with a horizontal directionality with an average of 16.5 pixels per second for Figure S3C and 18 pixels per second for Figure S3D.

Next to the effect of imaging directionality using a continuous rastering acquisition mode, we evaluated the effect of imaging speed (i.e. in scans per second) on the image quality achievable and effect on sensitivity. Fig. 3 shows the results of cross-sectional imaging runs at scan rates ranging from 5 to 40 scans per second (with 1 pixel $=1$ scan). Fig. 3B shows extracted spectra for each image in Fig. 2A. Whilst the spectrum quality is maintained, some loss of intensity occurs at higher scan rates. This is a consequence of a reduction in the number of laser shots per pixel, and a decreasing duty cycle due to the fixed interscan delay (i.e. time between two TOF events / scans).

Additional experiments were conducted to assess the performances of the UMALDI design combined with fast raster imaging in the negative ion mode at both 50 and $20 \mu \mathrm{m}$ spatial resolutions using norharmane as matrix. However, we observed a significant loss of signal when combining continuing raster acquisition at high speed (20 pixels per second) with this particular matrix. We hypothesize that the time the energy required for thermal desorption maybe significantly lower than the laser energy required for ionization for norharmane compared to CHCA, for which we did not observed a significant loss in signal (Figure S4c). As such the localized heating effect at the leading edge is evaporating the matrix before ionization can occur. Additional experiments demonstrated that a higher laser power was needed to reach the ionization threshold with norharmane compared to CHCA. We also notice a more significant increase in the laser ablation area with an increase of laser power for norharmane compared to CHCA (Figure S4a,b), which could ultimately create oversampling and thus create the loss of signal observed. Future studies could be directed to evaluate different matrices and their suitability with rapid continuous raster acqusition mode in MSI.

Therefore, we performed our further experiments in negative ion mode on consecutive mouse brain samples sprayed with CHCA and using a scan rate of 20 pixels per second in order to assess the image quality associated to speed to a bigger sample. Both 50 and $20 \mu \mathrm{m}$ spatial resolutions were compared and measured using the uMALDI source (Fig. 4A and 4B). The average intensity for both experiments was comparable which is showing that it is possible to play with the spatial resolution without affecting the average signal for lipids. For the half mouse brain imaged at $20 \mu \mathrm{m}$ spatial resolution, 511467 pixels were composing the region of interest corresponding to a surface of $204.6 \mathrm{~mm}$ [2]. The measurement took $8 \mathrm{~h}$ and $55 \mathrm{~min}$.

With the same parameters, a $50 \times 50 \mu \mathrm{m}^{2}$ pixel size measurement has been performed. In that case, 76,660 pixels (191 mm [2]) were imaged in $3 \mathrm{~h}$ and $40 \mathrm{~min}$. The image acquired with pixel size of $20 \times 20 \mu \mathrm{m}^{2}$ was closer to the morphological structures of the brain tissue as revealed by the H\&E staining (Fig. 4B). Nevertheless, we noticed a change in the ratio that is observable when looking at the scale intensities of Figs. 4A and 4B between $\mathrm{m} / \mathrm{z} 906.60$ and the two other species which is observable in the single spectra obtained from the same area of the brain (cortex). It is also interesting to notice that the $\mathrm{S} / \mathrm{N}$ in general was higher when running the $50 \times 50 \mu \mathrm{m}^{2}$ experiment for the lipids between $\mathrm{m} / \mathrm{z} 800$ and 850 (Fig. 4D and E). That could be explained by the fact that the laser beam was bigger at $50 \mu \mathrm{m}$ (unfocused) leading to an easier extraction for the less abundant species. Recently, some publications have shown that analytes can migrate through the matrix layer using SIMS technology which can explain why the ratio changed. Indeed, when shooting with a $15 \mu \mathrm{m}$ laser beam, more shots in one location are expected than at $50 \mu \mathrm{m}$ leading to depth of matrix profiled. $[22,23]$

\subsection{High spatial resolution MALDI-MSI of cartilage tissue}

Cartilage is composed of $10-30 \%$ collagen, $3-10 \%$ proteoglycans and other glycoproteins and lipids. Proteoglycans and collagen are interacting with each other establishing a strong network allowing pressure resistance to loadings and osmotic properties [24]. Only one type of cell is present in the cartilage and called chondrocytes.

It has been shown that lipids are mainly produced by the chondrocytes and play an important role in cartilage metabolism despite their low concentration within human cartilage [25]. Cillero-Pastor et al. have previously described the differential lipid distribution in human healthy and osteoarthritic cartilage by time 

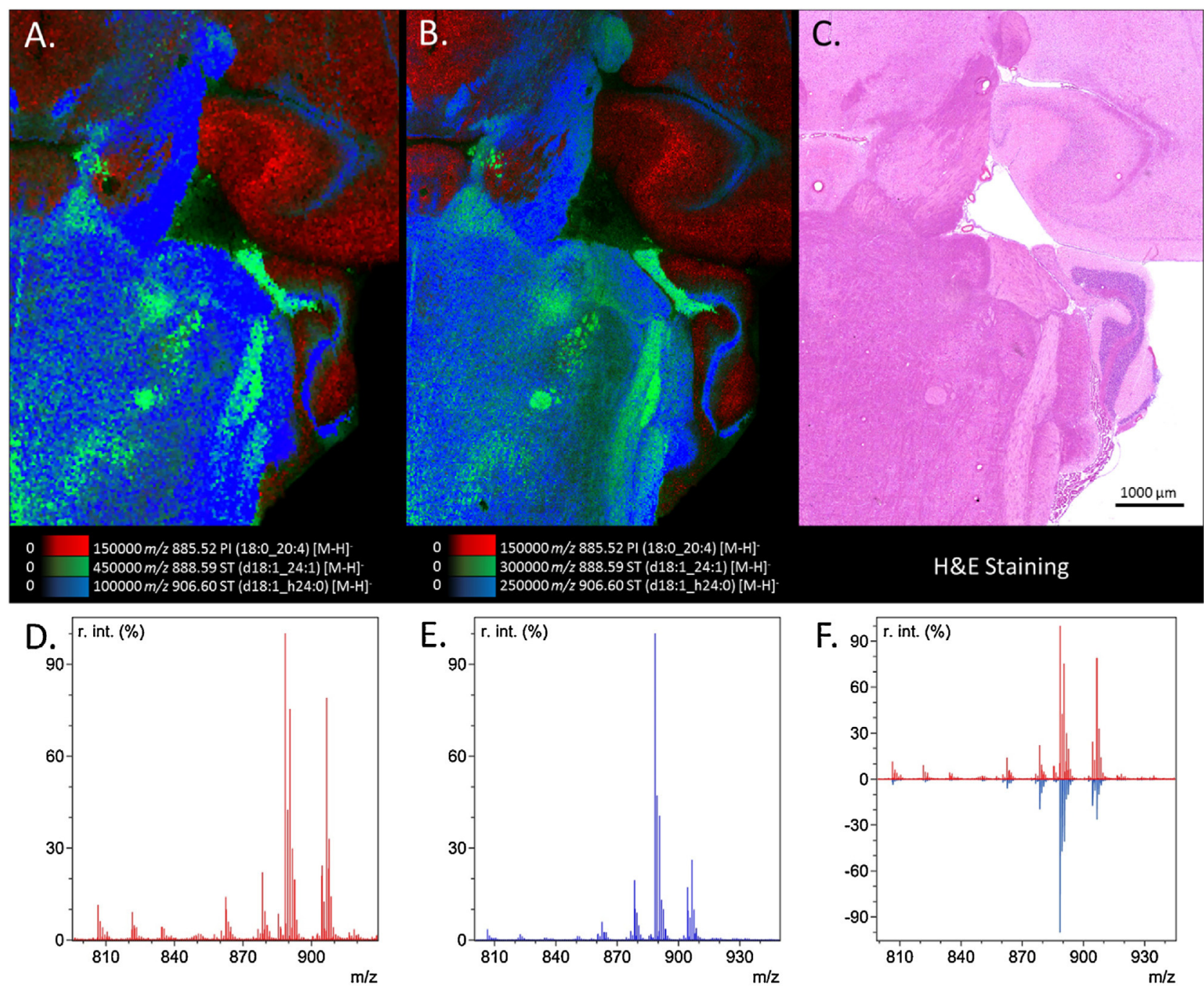

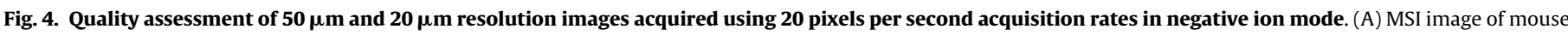

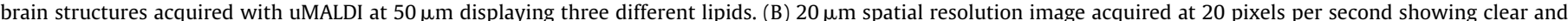

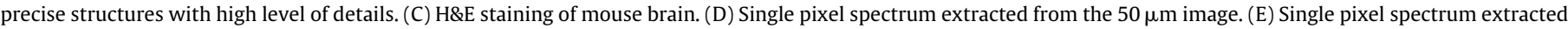

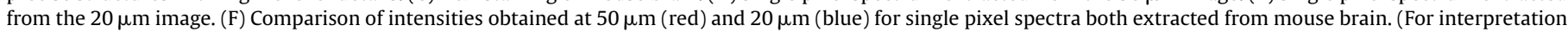
of the references to colour in this figure legend, the reader is referred to the web version of this article.)

of flight secondary ion mass spectrometry instruments (TOF-SIMS) [26]. Unfortunately, TOF-SIMS lacks sensitivity for the detection of molecules over $m / z 1000$ and low acquisition rates compared to MALDI. In this work, we show for the first time the lipid distribution of human OA cartilage at cellular level by employing the uMALDI source operated at $15 \mu \mathrm{m}$ spatial resolution. Fig. 5A shows a Safranin-O staining slide combined with Fast-Green without any MSI performed of the tissue revealing that OA cartilage tissue sections are mainly composed of proteoglycans (red colored tissue with Safranin-O) and showing that only few cells are present in OA sections (nuclei chondrocytes are stained in blue with hematoxylin). uMALDI-MSI experiments have been performed on a consecutive section at $15 \mu \mathrm{m}$ spatial resolution using continuing raster mode at a speed of 20 pixels / seconds (Fig. 5B). The extracted ion images display three main and distinct regions within $\mathrm{OA}$ tissue that can be linked to histology; first, the superficial part of the cartilage matrix as shown in red and based on the distribution of the ion $m / z$ 703.57, identified as protonated species of sphingomyelin [SM (d18:1_16:0)+H] $]^{+}$. Secondly, the ion $m / z 746.57$ identified as protonated specie of DMPE (34:1) is predominantly present in cartilage matrix (shown in blue). Finally and more interestingly, we were able to link the presence of $m / z 810.60\left(\left[\mathrm{PC}\left(18: 0 \_18: 1\right)+\mathrm{Na}\right]^{+}\right)$ to chondrocyte cluster cells displayed in yellow and validated with the staining image of the acquired section (Fig. 5C). In addition, this stained section after MALDI-MSI acquisition showing that the laser shot all over the tissue and let line marks spaced of more or less $11 \mu \mathrm{m}$ allowing us to get signal from all the chondrocyte cells despite their small size (10 to $30 \mu \mathrm{m}$ ) and poor abundance in OA tissue. This is due to the high laser energy we had to use to get signal from the cartilage tissue. Using uMALDI, we are now able to reach the high spatial resolution on cartilage sections needed to investigate the micro-environment of the chondrocytes.

\subsection{Ion mobility imaging at $20 \mu \mathrm{m}$ spatial resolution at 10 scans per second}

We further assessed the capability of the platform for performing ion mobility separation of isobaric lipid species at $20 \mu \mathrm{m}$ later resolution and high acquisition rate. Data were acquired in rastering mode at 10 scans per second. An example is given Fig. 6 where MALDI-ion mobility-MS imaging data were acquired at in a rat brain cross section. In the data set, we observed two isobaric ion species: $\left[\mathrm{PC}\left(18: 1 \_18: 0\right)+\mathrm{K}\right]^{+}(\mathrm{m} / z$ 826.60) and an isobaric species at $m / z$ 826.47. The extracted ion mobilogram for each ion species 


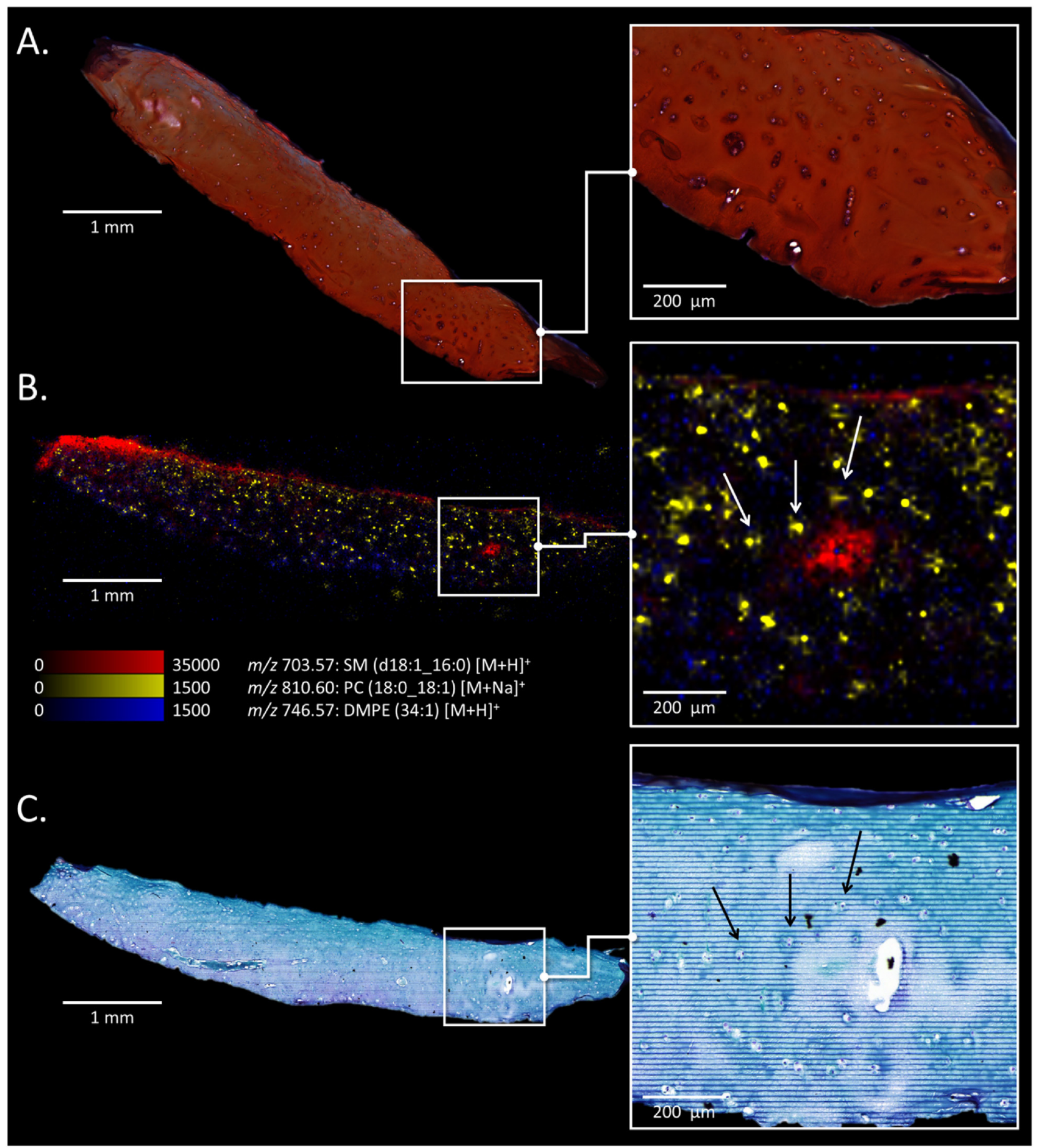

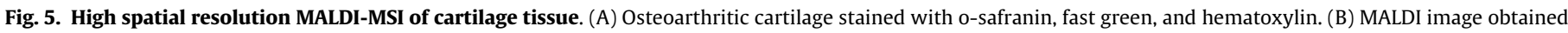

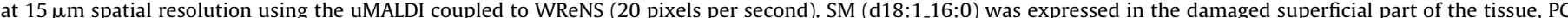

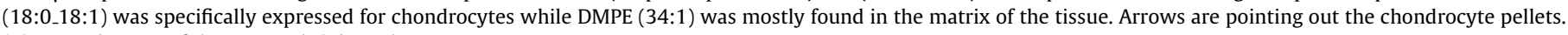
(C) Stained image of the acquired slide with continuing raster.

revealed distinct drift times (dt) for the two species (Fig. $6 \mathrm{~A}-\mathrm{C}$ ); namely $\mathrm{dt}=135$ (bins) for $m / z 826.47$ and $\mathrm{dt}=144$ (bins) for $m / z$ 826.60. This demonstrates that the ion mobility separation was maintained for the two isobaric species, even at unprecedented acquisition rates [27,28]. The reconstructed MALDI-ion mobility MS images display for each ion at their respective drift time different distributions of the selected ion species within the samples. The advantage of adding ion mobility separation after surface sam- pling such as MALDI and high resolution TOF-MS, is the additional selectivity provided when analyzing complex biological samples without chromatographic separation.

In addition, we noticed some minor aliasing (or phasing) in the reconstructed ion images; which is illustrated by a distortion of the ion image generated (Figure S5). The aliasing or phasing effect is depending on the transfer T-wave velocity, pusher frequency and mass range. When there is a mismatch between the frequency of 

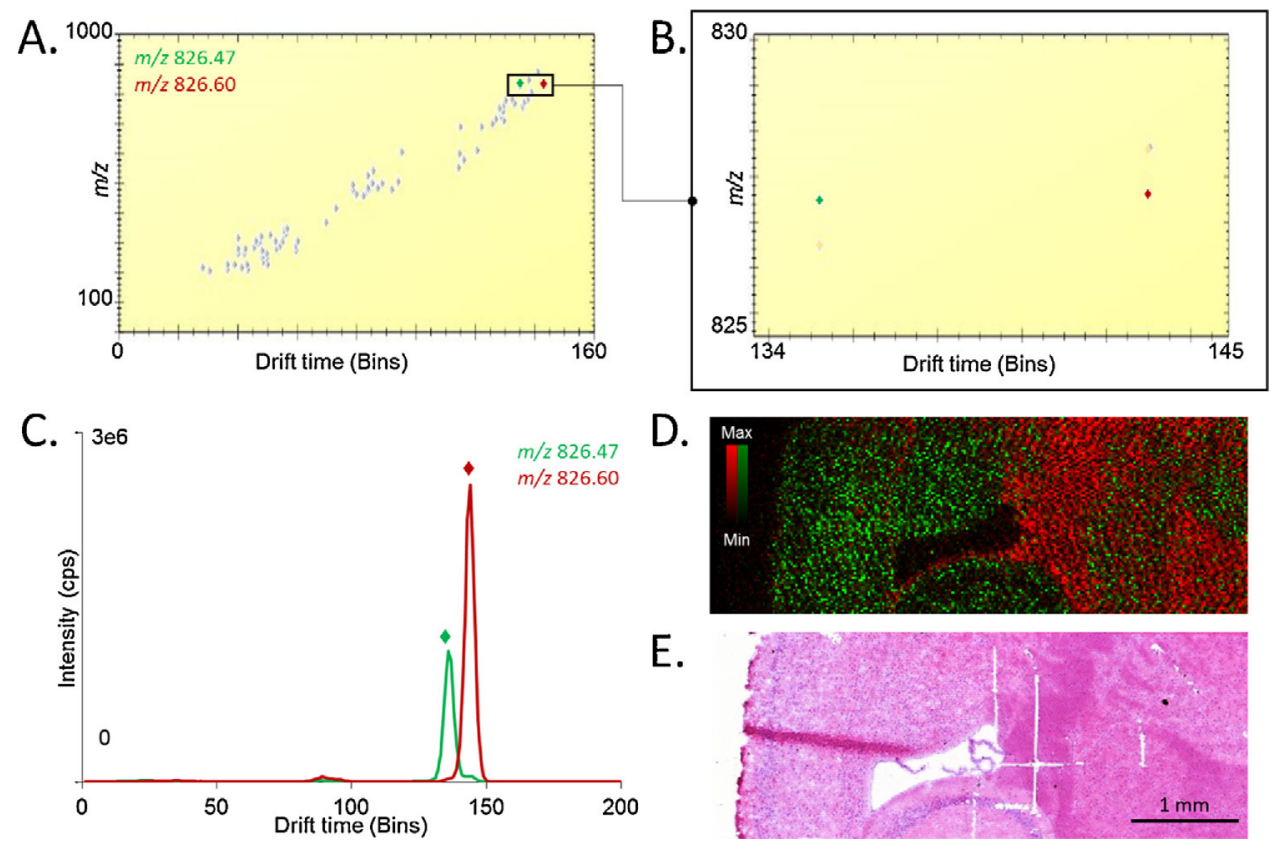

E.

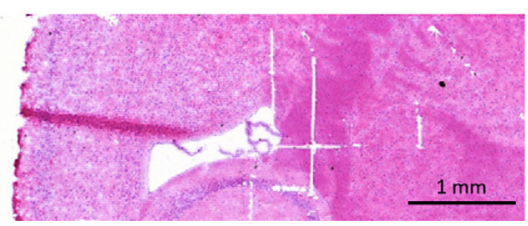

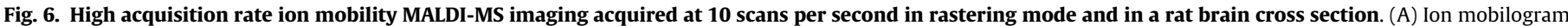

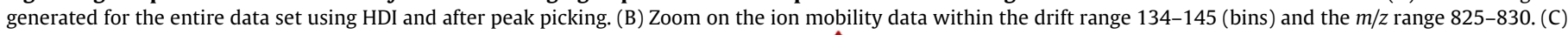

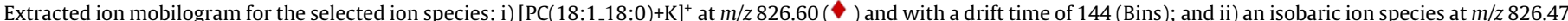

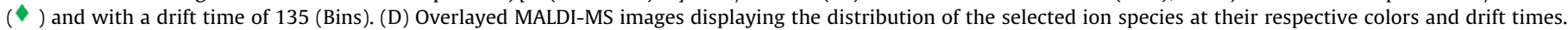

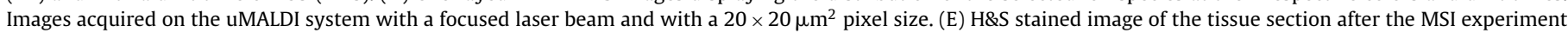

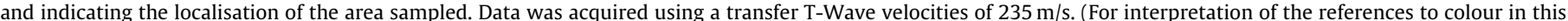
figure legend, the reader is referred to the web version of this article.)

arrival of the ion packets at the TOF pusher (which in turn is sampling at a frequency that is dependent on the TOF mass range), this results in an aliasing effected. We demonstrate that a careful optimization of the transfer T-wave velocity is critical in continuous rastering mode to obtain ion mobility-based MS images (Figure S6). For $m / z$ up to 1000 , we successfully maintained the ion mobility separation (see Figure S7) with minor changes in the drift time for selected species and limiting the aliasing by using a transfer TWave velocity of $235 \mathrm{~m} / \mathrm{s}$ (Fig. 6). This makes this platform unique for ion mobility imaging with fast acquisition rates and high spatial resolution.

\section{Conclusion}

The present work describes an improved design of a MALDI source mounted on a SYNAPT HDMS G2-Si mass spectrometer. Here, we demonstrate the improved performances of the new uMALDI source for lipid imaging in tissue sections down to a lateral resolution of $15 \mu \mathrm{m}$ without oversampling. In addition, the improvement in the design of the source considerably facilitates the handling and reduces instrument downtime compared to the previous version. This significantly improves the image quality not only in terms of lateral information but also for the overall increased sensitivity.

We also introduce a new web-based MS control program (i.e. "WREnS") which enables raster imaging and increases the analytical throughput. While analysis with the first generation system is performed with acquisition rates of a maximum of 8 pixel(s) per second, the uMALDI allows acquisition rates up to 20 pixels per second - with high lateral resolution $(<20 \mu \mathrm{m})$ directly on tissue. The overall increase of acquisition speed enabled by the WREnS software offers great promises for conducting analysis of larger patient cohorts in clinical research for instance where high throughput is required.
We also demonstrate the capability of this platform to characterize the lipid distribution of osteoarthritic cartilage down to cellular level, which highlights the benefit for application in a broader clinical context. Finally, we prove that ion mobility separation of lipid species is maintained and images can be acquired at a rate of 10 scans per seconds with post-ionization separation. This renders a unique ion mobility mass spectrometric imaging platform with high acquisition rates and high spatial resolution. This paves the way for more in-depth and spatially resolved analysis of biological tissue samples where the separation of isobaric species, throughput and sensitivity become critical and broaden the scope of application.

\section{Acknowledgments}

The research was performed in the M4I research program that was financially supported by the Dutch Province of Limburg as part of the "LINK" program. Florian Barré received funding from the European Union's Horizon 2020 research and innovation program under the Marie Sklodowska-Curie Program, TargetCare (ITN-2014-ETN 642,414). Beatriz Rocha is funded by Xunta de Galicia (IN606B-2016/004). Rat brain tissue sections were kindly provided by Martin R.L. Paine (M4I) and Audrey Jongen (Department of General Surgery, Maastricht University Medical Center MUMC+). We acknowledge Dr. Laura Cremers (Orthopedics Department, University Medical Centre Utrecht (UMCU) to provide us with cartilage samples. We acknowledge Matthew Henderson (Waters) for excellent technical support.

\section{Appendix A. Supplementary data}

Supplementary material related to this article can be found, in the online version, at doi:https://doi.org/10.1016/j.ijms.2018.09. 015. 


\section{References}

[1] P. Chaurand, J. Proteomics 75 (2012) 4883-4892.

[2] D. Gode, D.A. Volmer, Analyst 138 (2013) 1289-1315.

[3] C.N. Ferguson, J.W. Fowler, J.F. Waxer, R.A. Gatti, J.A. Loo, Adv. Exp. Med. Biol. 806 (2014) 283-299.

[4] H. Gagnon, J. Franck, M. Wisztorski, R. Day, I. Fournier, M. Salzet, Prog. Histochem. Cytochem. 47 (2012) 133-174.

[5] K. Schwamborn, R.M. Caprioli, Nat. Rev. Cancer 10 (2010) 639-646.

[6] E.H. Seeley, R.M. Caprioli, Proc. Natl. Acad. Sci. U. S. A. 105 (2008) 18126-18131.

[7] S.R. Ellis, J. Cappell, N.O. Potocnik, B. Balluff, J. Hamaide, A. Van der Linden, R.M. Heeren, Analyst 141 (2016) 3832-3841.

[8] A. Zavalin, J. Yang, K. Hayden, M. Vestal, R.M. Caprioli, Anal. Bioanal. Chem. 407 (2015) 2337-2342.

[9] B. Balluff, M. Hanselmann, R.M. Heeren, Adv. Cancer Res. 134 (2017) 201-230.

[10] P.M. Vaysse, R.M.A. Heeren, T. Porta, B. Balluff, Analyst 142 (2017) 2690-2712.

[11] B. Cillero-Pastor, G.B. Eijkel, A. Kiss, F.J. Blanco, R.M. Heeren, Arthritis Rheum. 65 (2013) 710-720.

[12] M. Kriegsmann, R. Casadonte, T. Randau, S. Gravius, P. Pennekamp, A. Strauss, J. Oldenburg, K. Wieczorek, S.O. Deininger, M. Otto, J. Kriegsmann, Haemophilia 20 (2014) 446-453.

[13] B.M. Prentice, C.W. Chumbley, R.M. Caprioli, J. Mass Spectrom. 50 (2015) 703-710

[14] J.M. Spraggins, D.G. Rizzo, J.L. Moore, M.J. Noto, E.P. Skaar, R.M. Caprioli, Proteomics 16 (2016) 1678-1689.
[15] S. Da, Applied Biosystems Technical Note, 2008.

[16] N. Ogrinc Potocnik, T. Porta, M. Becker, R.M. Heeren, S.R. Ellis, Rapid Commun. Mass Spectrom. 29 (2015) 2195-2203.

[17] J.M. Spraggins, R.M. Caprioli, J. Am. Soc. Mass Spectrom. 22 (2011) 1022-1031.

[18] M. Sans, C.L. Feider, L.S. Eberlin, Curr. Opin. Chem. Biol. 42 (2017) 138-146.

[19] K. Sladkova, J. Houska, J. Havel, Rapid Commun. Mass Spectrom. 23 (2009) $3114-3118$

[20] J.C. Jurchen, S.S. Rubakhin, J.V. Sweedler, J. Am. Soc. Mass Spectrom. 16 (2005) 1654-1659.

[21] A. Rompp, B. Spengler, Histochem. Cell Biol. 139 (2013) 759-783.

[22] S. Van Nuffel, N. Elie, E. Yang, J. Nouet, D. Touboul, P. Chaurand, A. Brunelle, Anal. Chem. 90 (2018) 1907-1914.

[23] M.D. Pour, E. Jennische, S. Lange, A.G. Ewing, P. Malmberg, Sci. Rep. 6 (2016).

[24] N.P. Cohen, R.J. Foster, V.C. Mow, J. Orthop. Sports Phys. Ther. 28 (1998) $203-215$.

[25] A. Villalvilla, R. Gomez, R. Largo, G. Herrero-Beaumont, Int. J. Mol. Sci. 14 (2013) 20793-20808.

[26] B. Cillero-Pastor, G. Eijkel, A. Kiss, F.J. Blanco, R.M. Heeren, Anal. Chem. 84 (2012) 8909-8916.

[27] J. Stauber, L. MacAleese, J. Franck, E. Claude, M. Snel, B.K. Kaletas, I.M.V.D. Wiel, M. Wisztorski, I. Fournier, R.M.A. Heeren, J. Am. Soc. Mass Spectrom. 21 (2010) 338-347.

[28] K. Skraskova, E. Claude, E.A. Jones, M. Towers, S.R. Ellis, R.M.A. Heeren, Methods 104 (2016) 69-78. 